\title{
ESTUDIO TEÓRICO Y COMPUTACIONAL DE LA PIRÓLISIS DEL 4-CLORO-1-BUTANOL EN FASE GASEOSA MEDIANTE LA TEORÍA DEL FUNCIONAL DE LA DENSIDAD
}

\author{
THEORETICAL AND COMPUTATIONAL STUDY \\ OF THE PYROLYSIS OF 4-CHLORO-1-BUTANOL IN THE \\ GAS PHASE USING DENSITY FUNCTIONAL THEORY
}

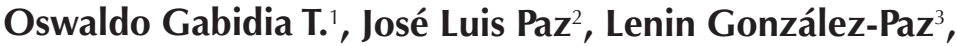 Edgar Márquez ${ }^{4}$, Joan Vera-Villalobos ${ }^{5}$, José Mora ${ }^{6}$, Ysaias Alvarado7 \& Marcos Loroño ${ }^{1 *}$}

Recibido: 27 de octubre 2021 / Aceptado: 5 de enero 2022

DOI 10.26807/ia.v10i1.225

1 Universidad Nacional Mayor de San Marcos, Facultad de Química e Ingeniería Química, Departamento Académico de Química Analítica e Instrumental, Lima, Perú. (oswaldo.gabidia@unmsm.edu.pe;

* correspondencia: mloronog@unmsm.edu.pe)

2 Universidad Nacional Mayor de San Marcos, Facultad de Química e Ingeniería Química, Departamento Académico de Química Inorgánica, Lima, Perú. (jpazr@unmsm.edu.pe)

3 Universidad del Zulia (LUZ), Departamento de Biología Facultad Experimental de Ciencias (F.E.C), Laboratorio de Genética y Biología Molecular (L.G.B.M), Maracaibo, República Bolivariana de Venezuela. (Igonzalezpaz@gmail.com)

4 Universidad del Norte, Facultad de Ciencias Exactas, Departamento de Química y Biología, Grupo de Investigaciones en Química y Biología, Barranquilla, Colombia. (ebrazon@uninorte.edu.co)

5 Escuela Superior Politécnica del Litoral, Facultad de Ciencias Naturales y Matemáticas, Guayaquil, Ecuador. (venaroja@gmail.com)

6 Universidad San Francisco de Quito, Departamento de Ingeniería Química, Grupo de Química Computacional y Teórica (QCT-USFQ), Quito, Ecuador. (jrmora@usfq.edu.ec)

7 Instituto Venezolano de Investigaciones Científicas (IVIC), Centro de Investigación y Tecnología de Materiales (CITeMA), Laboratorio de Caracterización Molecular y Biomolecular, Maracaibo, República Bolivariana de Venezuela. (alvaradoysaias@gmail.com) 
Palabras clave: 4-cloro-1-butanol, DFT, IBSI, mecanismo, $\mathrm{NCl}$, pirolisis. Keywords: 4-chloro-1-butanol, DFT, IBSI, mechanism, NCI, pyrolysis

\section{RESUMEN}

Se investigó el mecanismo y la cinética de la pirolisis en fase gaseosa del 4cloro-1-butanol a nivel DFT. La reacción da como productos tetrahidrofurano, formaldehido, propeno y cloruro de hidrogeno. El mecanismo de eliminación molecular sugiere dos vías de reacción. La primera vía se produce a través de un estado de transición cíclico de cuatro miembros, donde el nivel de teoría WB97XD/6-31G++(d,p) mostró resultados cercanos con los parámetros cinéticos experimentales. La segunda vía ocurre en dos etapas, la primera se da mediante un estado de transición cíclico de cuatro miembros y la segunda con estado de transición cíclico de seis miembros, los niveles de teoría que dieron resultados más cercanos a los experimentales fueron B3LYP/6-31G(d,p) y WB97XD/6-31G++(d,p), para primera y segunda etapa respectivamente. La ruptura del enlace $\mathrm{C}-\mathrm{Cl}$, debido a su polarización en el enlace, es el paso determinante de la velocidad en ambas vías de reacción. El análisis topológico $\mathrm{NCl}$ se usó para determinar las interacciones no covalentes es todas las estructuras. La variación de la fuerza de los enlaces involucrados en las transiciones se midió mediante el índice IBSI, lo que permitió verificar el mecanismo propuesto.

\section{ABSTRACT}

The mechanism and kinetics of gas-phase pyrolysis of 4-chloro-1-butanol at DFT level were investigated. The reaction gives as products tetrahydrofuran, formaldehyde, propene and hydrogen chloride. The molecular elimination mechanism suggests two reaction pathways. The first pathway occurs through a four-member cyclic transition state, where the theory level WB97XD/6$31 \mathrm{G}++(\mathrm{d}, \mathrm{p})$ showed results close to experimental kinetic parameters. The second pathway occurs in two stages, the first is given by a cyclic transition state 
of four members and the second with a cyclical transition state of six members, the levels of theory that gave results closest to the experimental ones were B3LYP/6-31G $(d, p)$ and WB97XD/6-31G++(d,p), for first and second stage respectively. The breakdown of the $\mathrm{C}-\mathrm{Cl}$ bond, due to its polarization at the bond, is the velocity-determining step in both reaction pathways. The $\mathrm{NCl}$ topological analysis was used to determine the non-covalent interactions of all structures. The variation of the strength of the bonds involved in the transitions was measured by the IBSI index, to verify the proposed mechanism.

\section{INTRODUCCIÓN}

El estudio sobre la pirolisis y la deshidrogenación de haluros de alquilo ha sido estudiado por diversos autores (Maccoll, 1969; Smith et al., 1971; Chuchani, 1995), en ellos se plantea un estado de transición cíclico concertado de cuatro miembros con la formación de su correspondiente olefina, según se muestra en el Esquema 1.

En este planteamiento la presencia de un $\beta$-hidrógeno adyacente al enlace $C-X$ es necesario para realizar la transición.

El estudio sobre la pirolisis del 4-metoxi-1-clorobutano mostraron que el grupo ayuda anquiméricamente en la deshidrogenación, para dar como producto tetrahidrofurano, lo que sugiere un mecanismo de par iónico íntimo, este resultado se considera una evidencia de la participación de grupos vecinos en la pirolisis en fase gaseosa de ciertos haluros de alquilo (Chuchani et al., 1986).

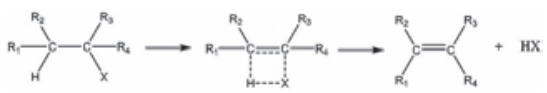

Esquema 1. Mecanismo de eliminación de haluros de alquilo

Maccoll y Thomas propusieron que este mecanismo se lleva a cabo debido a la polarización del enlace $\mathrm{C}-\mathrm{X}\left(\mathrm{C}^{\delta+} \ldots \mathrm{X}^{\delta_{-}}\right)$, con la participación del enlace $\mathrm{C}-\mathrm{H}$ adyacente (Maccoll et al., 1955). Luego estos mismos au- 
tores propusieron un mecanismo de estado de transición polar con la formación de un par iónico íntimo y presentaron varias evidencias para apoyar esta idea (Maccoll, 1967).

Para tal fin, el trabajo experimental estuvo a cargo de Chuchani y Martin (Chuchani et al., 1987), en la cual se estudió la cinética de eliminación por pirolisis del 4-cloro-1-butanol en fase gaseosa en un sistema estático. La reacción procedió en forma ho- mogénea y unimolecular, y se planteó un mecanismo de dos vías que pudiese explicar lo productos formados: tetrahidrofurano, formaldehido, propeno y cloruro de hidrogeno, según se observa en el Esquema 2.

A través de este estudio se evaluó la participación anquimérica del grupo $\mathrm{OH}$ y la ruptura del enlace $\mathrm{C}-\mathrm{Cl}$, a través de la formación de un par iónico intimo $\left(\mathrm{C}^{\delta+} \ldots \mathrm{X}^{\delta-}\right)$.

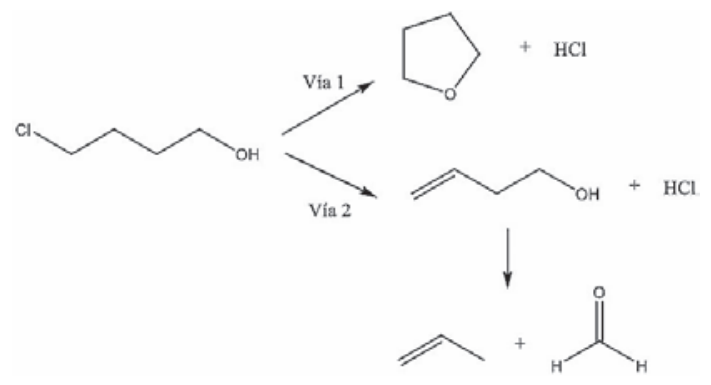

Esquema 2. Mecanismo general para la pirolisis del 4-cloro-1 butanol

\section{METODOLOGÍA COMPUTACIONAL}

El mecanismo y la cinética de la pirolisis del 4-cloro-1-butanol se estudió con el método DFT. Las estructuras y las frecuencias vibracionales armónicas de los reactivos, estados de transición y productos se optimi- zaron con los funcionales B3LYP, B3PW91, MPW1PW91, PBEPBE, WB97XD, B97D3. En cada caso se utilizó la base 6-31G, agregando funciones difusas y polarizadas. Estos cálculos se realizaron con el pro- 
grama Gaussian 16 (Gaussian 16, 2016), con los parámetros por defecto para la optimización de la geometría.

Se confirmaron las estructuras de los estados de transición con un análisis de frecuencias (una sola frecuencia imaginaria), luego se les realizó cálculos de coordenadas de reacción intrínsecas (IRC) para obtener perfiles de reacción completos. La energía vibracional de punto cero (ZPVE), las correcciones de temperatura y las entropías absolutas se determinaron mediante un análisis frecuencial. En los cálculos de frecuencias se utilizaron los promedios experimentales de la temperatura y presión. Los factores de escala para las frecuencias se tomaron de las referencias encontradas (Foresman et al., 1996; Scale Factors, 2021).

Se empleó la ecuación de Arrhenius y la de Eyring-Polanyi para determinar los coeficientes de velocidad y el cambio de energía libre de Gibbs entre el reactivo y el estado de transición $\left(\Delta \mathrm{G}^{\ddagger}\right)$, asumiendo un coeficiente de transición, $\alpha$, igual a uno de acuerdo con las siguientes ecuaciones:

$$
\begin{gathered}
k=A e^{\left(E_{a} / R T\right)} \\
k=\alpha\left(\frac{k_{B} T}{h}\right) e^{\left(\frac{-\Delta G^{\ddagger}}{R T}\right)} \\
\Delta G^{\neq}=\Delta H^{\neq}-T \Delta S^{\neq}
\end{gathered}
$$

Para determinar la energía de activación se empleó la siguiente ecuación para reacciones en fase gaseosa:

$$
E_{n}=\Delta H^{\ddagger}+m R T .
$$

Donde para una reacción unimolecular $\mathrm{m}=1$ y para reacciones biomoleculares $\mathrm{m}=2$.

Mediante el análisis poblacional de orbitales naturales de enlace (NBO), implementado en Gaussian 16 (NBO, 2016), se obtuvieron los índices de Wiberg (Wilber, 1968) que sirvieron para estudiar la evolución de la reacción a lo largo de las coordenadas implicadas en la formación de los productos. La ruptura y formación de enlaces implicados en el mecanismo de reacción son descritos utilizando el parámetro de sincronicidad $\left(S_{y}\right)$ (Moyano et al., 1989) definido por la ecuación:

$$
S_{y}=1-\frac{\left[\sum_{i=1}^{n} \frac{\left|\delta B_{i}-\delta B_{a v}\right|}{\delta B_{a v}}\right]}{2 n-2}
$$

donde $\mathrm{n}$ es el número de enlaces que intervienen directamente en la 
reacción y la variación relativa del índice de enlace se obtuvo de,

$$
\delta B_{i}=\left[B_{i}^{T S}-B_{i}^{R}\right] /\left[B_{i}^{P}-B_{i}^{R}\right.
$$

donde los superíndices $\mathrm{R}, \mathrm{TS}, \mathrm{P}$, representan respectivamente el reactivo, estado de transición y producto. Con este parámetro también se pudo calcular la evolución de cambio en el enlace durante la reacción como:

$$
\% E_{v}=\delta B_{i} \times 100
$$

Además, el valor medio requerido para el cálculo de la sincronicidad se calculó a partir de:

$$
\delta B_{a v}=1 / n \sum_{i=1}^{n} \delta B_{i}
$$

La topología molecular de interacciones no covalentes intramoleculares se determinó con el programa NCIplot (Contreras, 2011), donde se grafica el gradiente de la densidad reducida contra el signo del segundo autovalor propio de la matriz Hes- siana multiplicada por la densidad total de electrones, que genera un gráfico en 2D mostrando picos de interacción. las interacciones no covalentes también se observaron como isosuperficies en gráficos en 3D, donde se usó el programa VMD (Visual Molecular Dynamics, 2021) para poder visualizarlas.

La variación en la fuerza de enlace de los átomos involucrados en el mecanismo de reacción se determinó mediate el programa IGMplot (Klein et al., 2020), el cual usa el índice de fuerza de enlace intrínseco (IBSI) para sondear internamente la fuerza de un determinado enlace químico en situación molecular. De manera alternativa se usó el programa Multiwfn (Tian et al., 2012) para realizar los cálculos de interacciones no covalentes y para el cálculo del índice de fuerza de enlace intrínseco.

\section{RESULTADOS}

La Tabla 1 muestra los resultados de los parámetros termodinámicos y cinéticos calculados para la primera vía de la reacción y se comparan los resultados experimentales a 713,15 K. Los resultados se presentan para funcionales B3LYP, B3PW91, MPW1, PBE y posibles combinaciones. Todos estos métodos incluyeron correcciones por dispersiones empíricas y se compararon con los WB97XD y W97D3 que ya los incluían. 
Tabla 1. Parámetros termodinámicos y cinéticos calculados para la primera vía de reacción en la pirolisis del 4-cloro-1butanol en fase gaseosa a $440{ }^{\circ} \mathrm{C}(713,15 \mathrm{~K})$

\begin{tabular}{lccccc}
\hline Método & $\begin{array}{c}\mathbf{E}_{\alpha} \\
(\mathrm{kJ} / \mathrm{mol})\end{array}$ & $\begin{array}{c}\log \mathbf{A} \\
\left(\mathrm{s}^{-1}\right)\end{array}$ & $\begin{array}{c}\Delta \mathbf{H}_{\xi^{\beta}} \\
(\mathrm{kJ} / \mathrm{mol})\end{array}$ & $\begin{array}{c}\Delta \mathbf{S}^{\neq} \\
(\mathrm{J} / \mathrm{K} . \mathrm{mol})\end{array}$ & $\begin{array}{c}\Delta \mathbf{G}^{\neq} \\
(\mathrm{kJ} / \mathrm{mol})\end{array}$ \\
\hline Experimental & 231,3 & 13,78 & 225,4 & 2,94 & 223,0 \\
\hline B3LYP/6-31G & 142,3 & 16,0 & 136,36 & 46,4 & 96,14 \\
\hline B3LYP/6-31G d & 180,5 & 12,3 & 174,55 & $-25,9$ & 192,49 \\
\hline B3LYP/6-31G (d,p). & 177,0 & 12,2 & 171,03 & $-26,5$ & 189,93 \\
\hline B3LYP/6-31G ++(d,p). & 184,7 & 12,3 & 178,76 & $-25,9$ & 197,25 \\
\hline B3PW91/6-31G & 150,6 & 12,2 & 144,64 & $-27,4$ & 164,20 \\
\hline B3PW91/6-31G d & 190,1 & 12,2 & 184,20 & $-26,0$ & 202,74 \\
\hline B3PW91/6-31G (d,p). & 186,7 & 12,2 & 180,75 & $-26,8$ & 199,83 \\
\hline B3PW91/6-31G ++(d,p). & 193,7 & 12,2 & 187,74 & $-26,5$ & 206,63 \\
\hline MPW1PW91/6-31G & 165,6 & 12,2 & 159,72 & $-26,4$ & 178,55 \\
\hline MPW1PW91/6-31G d & 206,4 & 12,3 & 200,48 & $-25,0$ & 218,27 \\
\hline MPW1PW91/6-31G (d,p). & 203,0 & 12,3 & 197,03 & $-25,5$ & 215,20 \\
\hline MPW1PW91/6-31G ++(d,p). & 209,6 & 12,3 & 203,62 & $-25,0$ & 221,45 \\
\hline PBEPBE/6-31G & 122,8 & 12,1 & 116,9 & $-28,4$ & 137,1 \\
\hline PBEPBE/6-31G d & 159,4 & 12,2 & 153,5 & $-26,8$ & 172,6 \\
\hline PBEPBE/6-31G (d,p). & 156,3 & 12,2 & 150,4 & $-27,4$ & 169,9 \\
\hline PBEPBE/6-31G ++(d,p). & 166,1 & 12,2 & 160,2 & $-26,9$ & 179,4 \\
\hline WB97XD/6-31G d & 214,3 & 12,1 & 208,4 & $-29,5$ & 229,4 \\
\hline WB97XD/6-31G ++(d,p). & 216,7 & 12,0 & 210,7 & $-30,9$ & 256,6 \\
\hline B97D3/6-31G d & 154,0 & 12,2 & 148,0 & $-26,8$ & 167,1 \\
\hline B97D3/6-31G ++(d,p). & 188,5 & 12,1 & 182,6 & $-28,9$ & 203,2 \\
\hline
\end{tabular}

Para la segunda vía, los resultados se mas 3 y 4 . muestran en las Tablas 2 y 3, esque-<smiles>OCCCCCl</smiles><smiles>CCCCCCC</smiles><smiles>C1CCOC1</smiles> 
<smiles>OCCCCCl</smiles><smiles>CC(C)C</smiles><smiles>C1CC2=C(CC2)O1</smiles><smiles>C1CC1</smiles><smiles>C=CCCO</smiles><smiles>C=CC</smiles><smiles>C=O</smiles><smiles>C1=CCOC=C1</smiles>

Esquema 4. Mecanismo planteado para la segunda vía de reacción

Tabla 2. Parámetros termodinámicos y cinéticos calculados para la primera etapa de la segunda vía de reacción en la pirolisis del 4-cloro-1 butanol en fase gaseosa a $440{ }^{\circ} \mathrm{C}(713,15 \mathrm{~K})$

\begin{tabular}{lccccc}
\hline Método & $\begin{array}{c}\mathbf{E}_{\alpha} \\
(\mathrm{kJ} / \mathrm{mol})\end{array}$ & $\begin{array}{c}\log \mathbf{A} \\
\left(\mathrm{s}^{-1}\right)\end{array}$ & $\begin{array}{c}\Delta \mathbf{H}^{\neq} \\
(\mathrm{kJ} / \mathrm{mol})\end{array}$ & $\begin{array}{c}\Delta \mathbf{S}^{\neq} \\
(\mathrm{J} / \mathrm{K} . \mathrm{mol})\end{array}$ & $\begin{array}{c}\Delta \mathbf{G}^{\neq} \\
(\mathrm{KJ} / \mathrm{mol})\end{array}$ \\
\hline Experimental & 211,6 & 12,3 & 205,7 & $-24,3$ & 223,0 \\
\hline B3LYP/6-31G & 181,4 & 12,5 & 175,4 & $-21,7$ & 190,9 \\
\hline B3LYP/6-31G d & 226,4 & 12,5 & 220,4 & $-20,3$ & 234,9 \\
\hline B3LYP/6-31G (d,p) & 211,0 & 14,0 & 205,1 & 6,9 & 200,2 \\
\hline B3LYP/6-31G ++(d,p) & 205,4 & 13,9 & 199,5 & 6,0 & 195,2 \\
\hline B3PW91/6-31G & 184,4 & 12,5 & 178,5 & $-20,6$ & 193,2 \\
\hline B3PW91/6-31G d & 230,7 & 12,6 & 224,8 & $-19,3$ & 238,6 \\
\hline B3PW91/6-31G (d,p) & 226,0 & 12,5 & 220,1 & $-20,2$ & 234,6 \\
\hline B3PW91/6-31G ++(d,p) & 213,3 & 13,9 & 207,4 & 6,3 & 202,9 \\
\hline MPW1PW91/6-31G & 192,1 & 12,7 & 186,1 & $-17,5$ & 198,6 \\
\hline MPW1PW91/6-31G d & 240,0 & 12,7 & 234,1 & $-16,4$ & 245,8 \\
\hline MPW1PW91/6-31G (d,p) & 235,0 & 12,7 & 229,1 & $-18,3$ & 242,1 \\
\hline MPW1PW91/6-31G ++(d,p) & 221,7 & 14,0 & 215,8 & 7,0 & 210,9 \\
\hline PBEPBE/6-31G & 169,5 & 12,4 & 163,5 & $-23,7$ & 180,4 \\
\hline PBEPBE/6-31G d & 204,0 & 14,1 & 198,0 & 9,2 & 191,5 \\
\hline PBEPBE/6-31G (d,p) & 196,6 & 14,0 & 190,7 & 8,4 & 184,7 \\
\hline PBEPBE/6-31G ++(d,p) & 193,0 & 13,9 & 187,1 & 6,5 & 182,4 \\
\hline WB97XD/6-31G d & 247,3 & 12,8 & 241,4 & $-15,8$ & 252,7 \\
\hline WB97XD/6-31G ++(d,p) & 227,8 & 13,7 & 221,4 & 2,3 & 220,3 \\
\hline B97D3/6-31G d & 197,0 & 14,0 & 191,1 & 8,0 & 185,3 \\
\hline B97D3/6-31G ++(d,p) & 186,4 & 13,9 & 180,5 & 6,0 & 176,2 \\
\hline & & & & &
\end{tabular}


Tabla 3. Parámetros termodinámicos y cinéticos calculados para la segunda etapa de la segunda vía de reacción en la pirolisis del 4-cloro-1butanol en fase gaseosa a $440{ }^{\circ} \mathrm{C}(713,15 \mathrm{~K})$

\begin{tabular}{lccccc}
\hline Método & $\begin{array}{c}\mathbf{E}_{\alpha} \\
(\mathrm{kJ} / \mathrm{mol})\end{array}$ & $\begin{array}{c}\log \mathbf{A} \\
\left(\mathrm{s}^{-1}\right)\end{array}$ & $\begin{array}{c}\Delta \mathbf{H} \wedge \neq \\
(\mathrm{kJ} / \mathrm{mol})\end{array}$ & $\begin{array}{c}\Delta \mathbf{S} \wedge \neq \\
(\mathrm{J} / \mathrm{K} . \mathrm{mol})\end{array}$ & $\begin{array}{c}\Delta \mathbf{G} \wedge \neq \\
(\mathrm{kJ} / \mathrm{mol})\end{array}$ \\
\hline Experimental & 171,5 & 11,7 & 165,6 & $-37,5$ & 192,3 \\
\hline B3LYP/6-31G & 127,3 & 12,1 & 121,3 & $-29,5$ & 142,3 \\
\hline B3LYP/6-31G d & 130,2 & 12,2 & 124,3 & $-27,8$ & 144,1 \\
\hline B3LYP/6-31G (d,p) & 134,4 & 12,2 & 128,4 & $-26,8$ & 147,5 \\
\hline B3LYP/6-31G ++(d,p) & 142,5 & 12,2 & 136,5 & $-27,6$ & 156,2 \\
\hline B3PW91/6-31G & 125,5 & 12,0 & 119,5 & $-30,4$ & 141,2 \\
\hline B3PW91/6-31G d & 127,6 & 12,1 & 121,7 & $-28,8$ & 142,3 \\
\hline B3PW91/6-31G (d,p) & 132,3 & 12,2 & 126,4 & $-27,5$ & 145,9 \\
\hline B3PW91/6-31G ++(d,p) & 139,2 & 12,1 & 133,3 & $-28,2$ & 153,4 \\
\hline MPW1PW91/6-31G & 136,5 & 12,0 & 130,6 & $-31,0$ & 152,7 \\
\hline MPW1PW91/6-31G d & 137,8 & 12,1 & 131,9 & $-29,3$ & 152,8 \\
\hline MPW1PW91/6-31G (d,p) & 143,3 & 12,1 & 137,3 & $-28,0$ & 157,3 \\
\hline MPW1PW91/6-31G ++(d,p) & 150,2 & 12,1 & 144,3 & $-29,0$ & 165,0 \\
\hline PBEPBE/6-31G & 93,6 & 12,0 & 87,7 & $-30,5$ & 109,5 \\
\hline PBEPBE/6-31G d & 96,6 & 12,1 & 90,7 & $-29,4$ & 111,6 \\
\hline PBEPBE/6-31G (d,p) & 102,1 & 12,2 & 96,2 & $-27,4$ & 115,8 \\
\hline PBEPBE/6-31G ++(d,p) & 111,3 & 12,1 & 105,3 & $-28,5$ & 125,6 \\
\hline WB97XD/6-31G d & 150,6 & 12,1 & 144,7 & $-29,7$ & 165,8 \\
\hline WB97XD/6-31G ++(d,p) & 162,6 & 12,1 & 156,7 & $-29,1$ & 177,4 \\
\hline B97D3/6-31G d & 102,9 & 12,2 & 97,0 & $-26,5$ & 115,9 \\
\hline B97D3/6-31G ++(d,p) & 114,6 & 12,2 & 108,7 & $-27,7$ & 128,4 \\
\hline
\end{tabular}

Dichos resultados, fueron seleccionados exactamente bajo las mismas condiciones que la etapa 1 . De igual forma, se usaron diferentes funciones bases que rondaron desde 6$31 \mathrm{G}$ hasta incluir funciones de difusión $(++)$ y funciones de polarización que incluían a los átomos de hidrógenos. Los resultados se realizaron con diferentes métodos hasta encontrar que las energías calculadas fueran comparables con las experimentales, dentro de un $5 \%$ de error. Una veintena de cálculos por cada etapa fue necesaria. 
La Figura 1, muestra las variaciones de los cálculos energéticos con diferentes bases y métodos. Solo se muestra los resultados de la etapa determinante de la reacción; es decir, la primera etapa del primer compuesto. Los resultados claramente ponen en evidencia la divergencia de resultados alrededor de los resultados experimentales. No existe una clara correlación entre el uso de mejores métodos y bases y los resultados encontrados, en general si se agregan funciones de difusión, se mejoran los cálculos. Los métodos que incluyen MPW1PW91 se comparan con los WB97XD. En el primer caso, se tuvo que incluir dispersiones empíricas del tipo gd3bj, no así para el segundo caso (WB97XD) que ya las incluía. Para las demás etapas, figuras similares fueron añadidas en un material suplementario.

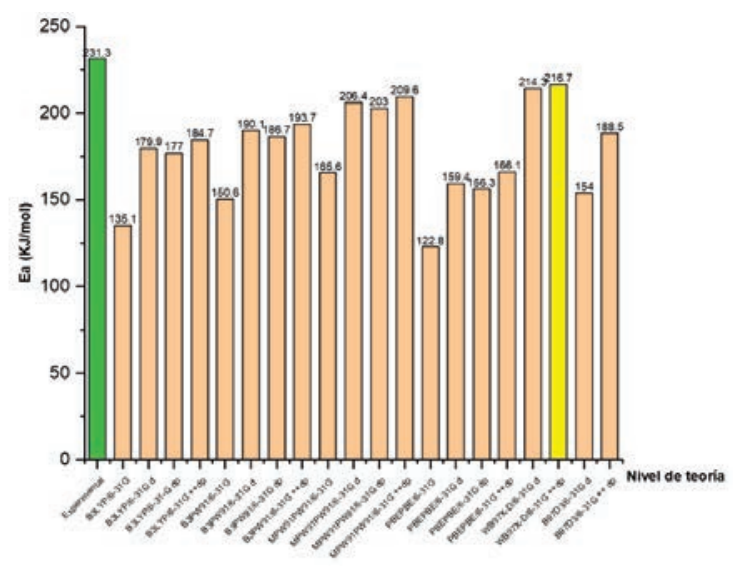

(a)

Figura 1. Comparación de la energía de activación experimental (verde) con los niveles de teoría empleados resaltando los más cercanos al experimental (amarillo), para (a) la primera vía de reacción, de reacción de la pirolisis del 4-cloro-1-butanol calculados a $440{ }^{\circ} \mathrm{C}$ 
De acuerdo con los esquemas 3 y 4 , en las Figuras 2-4 se pude observar los tres posibles estados de transiciones. La Figura 2, muestra un mecanismo con participación anquimérica del grupo $\mathrm{OH}$ y sus posibles productos de reacción.
En cambio, en la Figura 3, se vuelve a ejecutar la reacción sin participación del $\mathrm{OH}$. Finalmente, producto del esquema 4, se genera los compuestos también posibles en esta reacción (Figura 4).

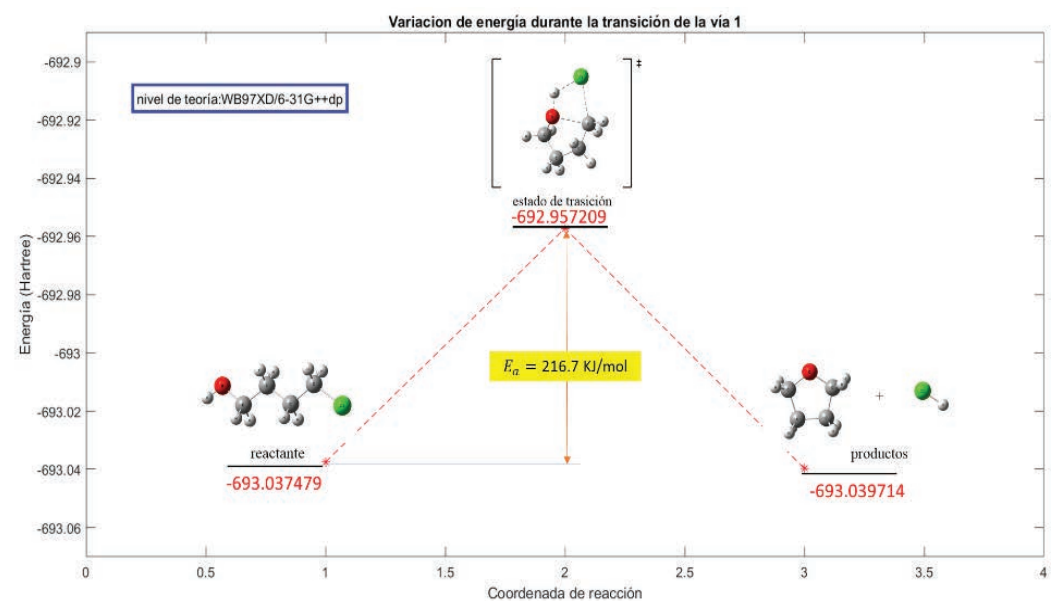

Figura 2. Variación de la energía para transición de la primera vía de reacción de la pirolisis del 4-cloro-1-butanol a $440{ }^{\circ} \mathrm{C}$. empleando el nivel de teoría WB97XD/6-31G++(d,p). 


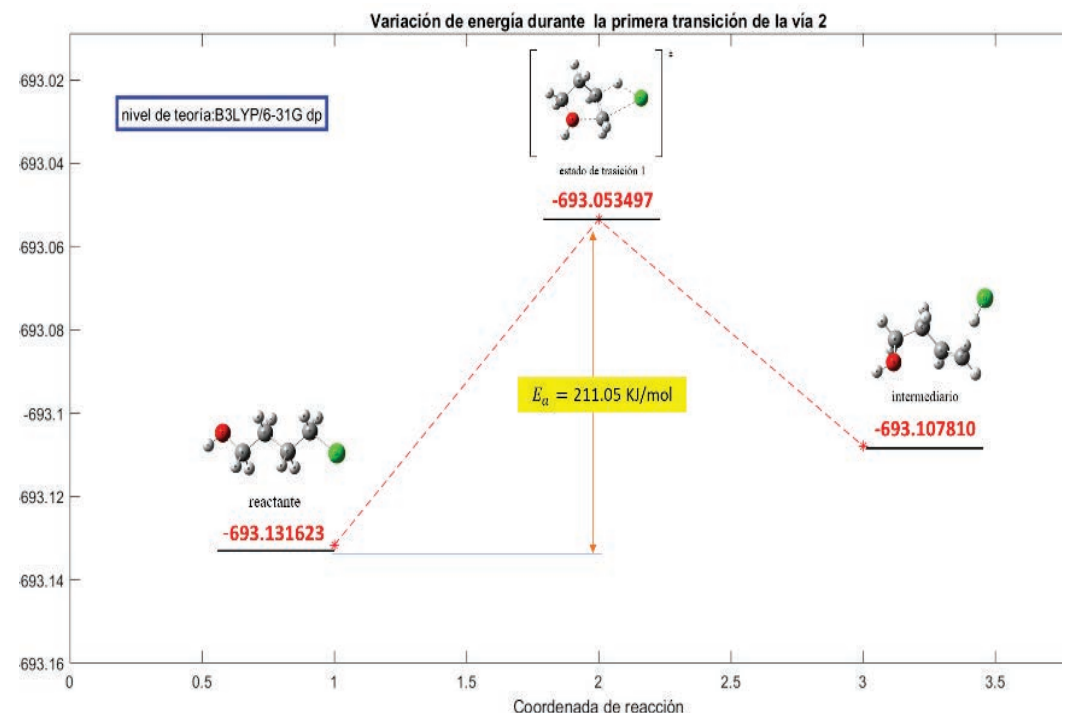

Figura 3. Variación de la energía para transición de la primera etapa de la segunda vía de reacción de la pirolisis del 4-cloro-1-butanol a $440^{\circ} \mathrm{C}$ empleando el nivel de teoría B3LYP/6-31G(d,p) 


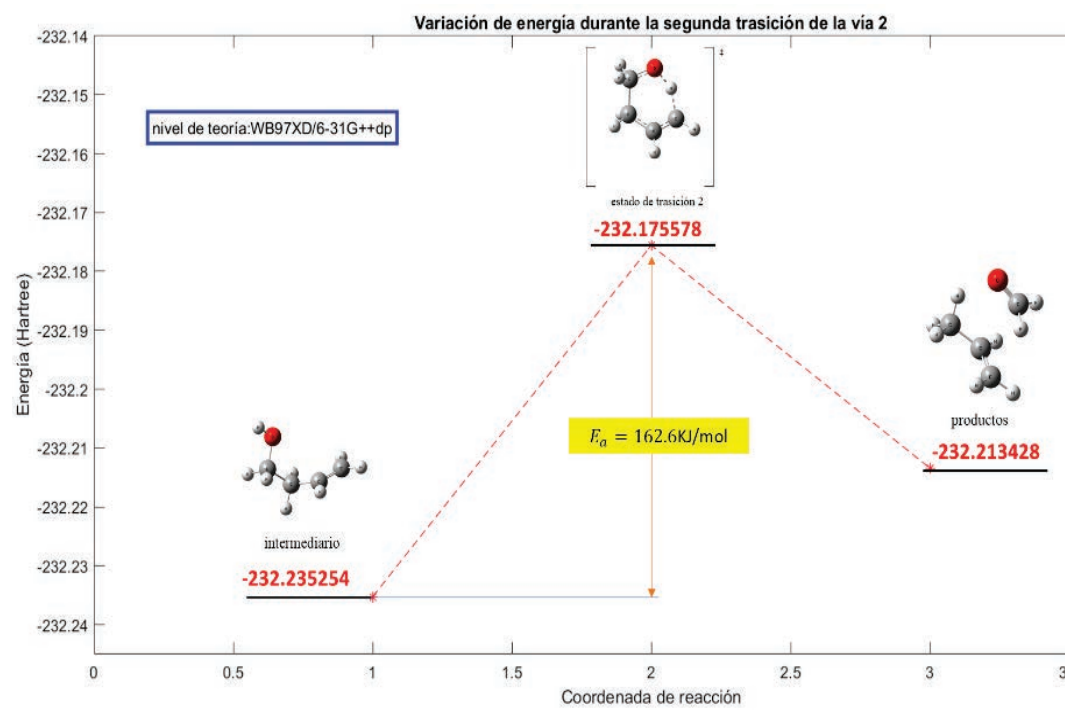

Figura 4. Variación de la energía para transición de la segunda etapa de la segunda vía de reacción de la pirolisis del 4-cloro-1-butanol a $440{ }^{\circ} \mathrm{C}$ empleando el nivel de teoría WB97XD/6-31G ++(d,p)

Las estructuras optimizadas para los reactivos $(R)$, estados de transición (TS) y productos $(\mathrm{P})$, para la vía 1, y la primera y segunda etapa de la vía 2 de la pirólisis del 4-cloro-1-butanol se muestran en la Figura 5. 
(a)

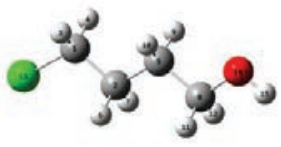

R

(b)

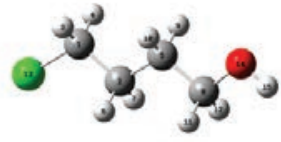

R

(c)

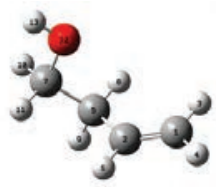

R

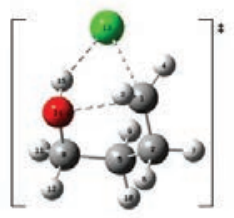

TS

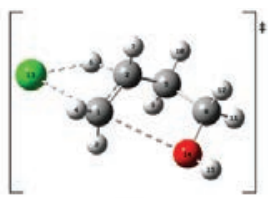

Ts

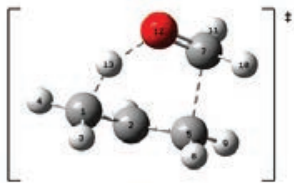

TS

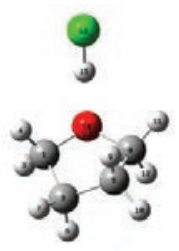

P

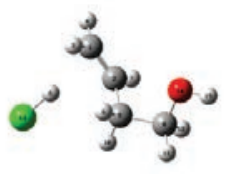

P

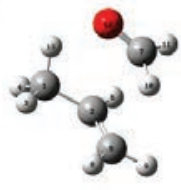

P

Figura 5. Estructuras optimizadas para el reactivo (R), estado de transición (TS) y productos $(P)$ en la primera vía de reacción (a), la primera etapa (b) y segunda etapa (c) de la segunda vía de reacción

En las Tablas 5 y 6 se muestran los parámetros optimizados del reacitvo (R), estado de transición (TS) y producto $(\mathrm{P})$ para la primera y segunda etapa de la segunda vía de reacción de la pirolisis del 4-cloro-1-butanol en fase gaseosa a $440{ }^{\circ} \mathrm{C}$, obtenidos con el nivel de teoría B3LYP/6-31G(d,p). 
Tabla 5. Parámetros estructurales del reactivo (R), estado de transición (TS)

y producto $(P)$ para la primera etapa de la segunda vía de reacción de la pirolisis del 4-cloro-1-butanol en fase gaseosa a $440{ }^{\circ} \mathrm{C}$, obtenidos con el nivel de teoría B3LYP/6-31G(d,p)

\begin{tabular}{|c|c|c|c|c|c|}
\hline \multicolumn{6}{|c|}{ Distancia atómica (Å) } \\
\hline & $\mathrm{Cl}_{13}-\mathrm{C}_{1}$ & $\mathrm{Cl}_{13}-\mathrm{H}_{6}$ & $\mathrm{C}_{1}-\mathrm{C}_{2}$ & $\mathrm{C}_{2}-\mathrm{H}^{6}$ & $\mathrm{O}_{14}-\mathrm{C}_{1}$ \\
\hline $\mathbf{R}$ & 1,82 & 2,93 & 1,52 & 1,10 & 4,89 \\
\hline TS & 2,69 & 1,84 & 1,40 & 1,25 & 2,81 \\
\hline $\mathbf{P}$ & 3,55 & 1,31 & 1,34 & 2,29 & 3,67 \\
\hline & \multicolumn{5}{|c|}{ Ángulos diedros (en grados) } \\
\hline & $\mathrm{C}_{1}-\mathrm{C}_{2}-\mathrm{H}_{6}-\mathrm{Cl}_{13}$ & $\mathrm{C}_{2}-\mathrm{H}_{6}-\mathrm{Cl}_{13}-\mathrm{C}_{1}$ & $\mathrm{H}_{6}-\mathrm{Cl}_{13}-\mathrm{C}_{1}-\mathrm{C}_{2}$ & $\mathrm{Cl}_{13}-\mathrm{C}_{1}-\mathrm{C}_{2}-\mathrm{H}_{6}$ & \\
\hline TS & $-5,13$ & 4,09 & $-1,86$ & 1,78 & \\
\hline & \multicolumn{5}{|c|}{ Frecuencia imaginaria $\left(\mathrm{cm}^{-1}\right)$} \\
\hline TS & 1288,91 & & & & \\
\hline
\end{tabular}

Tabla 6. Parámetros estructurales del reactivo (R), estado de transición (TS) y producto $(P)$ para la segunda etapa de la segunda vía de reacción de la pirolisis del 4cloro-1-butanol empleando el nivel de teoría WB97XD/6-31G++(d,p)

\begin{tabular}{|c|c|c|c|c|c|c|}
\hline \multicolumn{7}{|c|}{ Distancia atómica (Å) } \\
\hline & $\mathrm{C}_{5}-\mathrm{C}_{7}$ & $\mathrm{C}_{7}-\mathrm{O}_{12}$ & $\mathrm{O}_{12}-\mathrm{H}_{13}$ & $\mathrm{H}_{13}-\mathrm{C}_{1}$ & $\mathrm{C}_{1}-\mathrm{C}_{2}$ & \\
\hline $\mathbf{R}$ & 1,82 & 2,93 & 1,52 & 1,10 & 4,89 & \\
\hline TS & 2,69 & 1,84 & 1,40 & 1,25 & 2,81 & \\
\hline $\mathbf{P}$ & 3,55 & 1,31 & 1,34 & 2,29 & 3,67 & \\
\hline \multicolumn{7}{|c|}{ Ángulos diedros (en grados) } \\
\hline TS & $\begin{array}{c}C_{1}-C_{2}-C_{5}-C_{7} \\
-67,76\end{array}$ & $\begin{array}{c}\mathrm{C}_{2}-\mathrm{C}_{5}-\mathrm{C}_{7}-\mathrm{O}_{12} \\
47,52\end{array}$ & $\begin{array}{c}\mathrm{C}_{5}-\mathrm{C}_{7}-\mathrm{O}_{12}-\mathrm{H}_{13} \\
-24,69\end{array}$ & $\begin{array}{c}\mathrm{C}_{7}-\mathrm{O}_{12}-\mathrm{H}_{13}-\mathrm{C}_{1} \\
29,41\end{array}$ & $\begin{array}{c}\mathrm{O}_{12}-\mathrm{H}_{13}-\mathrm{C}_{1}-\mathrm{C}_{2} \\
-37,74\end{array}$ & $\begin{array}{c}\mathrm{H}_{13}-\mathrm{C}_{1}-\mathrm{C}_{2}-\mathrm{C}_{5} \\
58,88\end{array}$ \\
\hline \multicolumn{7}{|c|}{ Frecuencia imaginaria $\left(\mathrm{cm}^{-1}\right)$} \\
\hline TS & & & 1088,918 & & & \\
\hline
\end{tabular}

La Figura 6 muestra el resultado del cálculo IRC "Intrinsic Reaction Coordinate". En este proceso se pre- senta el esquema general de reacción desde el reactante a los productos, pasando por el estado de 
transición. El esquema es extrapolado hasta obtener los estados de mínimas energías, junto a dos posibles intermedios. El cálculo IRC repre- senta la primera etapa determinante de la reacción. Y se muestra un mecanismo con participación anquimérica.

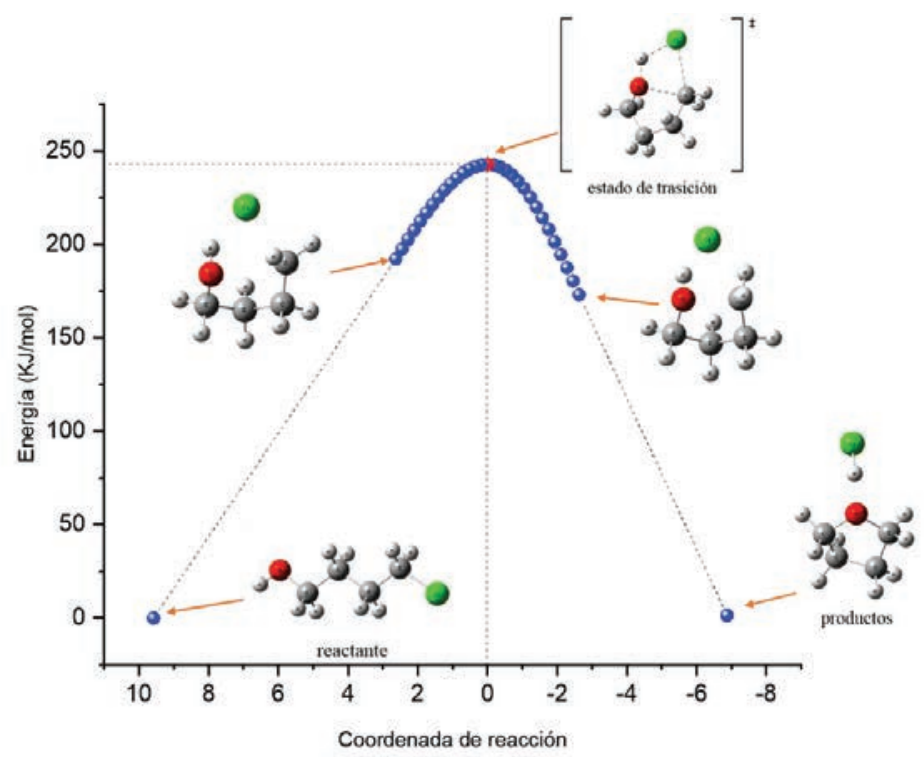

Figura 6. IRC para la primera vía de reacción de la pirolisis del 4-cloro-1-butanol empleando el nivel de teoría WB97XD/6-31G++(d,p)

La Figura 7, muestra el cálculo IRC de la segunda vía posible. En esta etapa, el mecanismo igual libera $\mathrm{HCl}$, pero sin formación de unión o enlace del grupo $\mathrm{OH}$.

En la Figura 8, se muestra el cálculo final IRC de la segunda etapa. Aquí, se obtienen los productos finales. Todos los cálculos fueron realizados con funcionales que incluyeran funciones de dispersión empíricas con el objeto de tomar en cuenta las posibles interacciones dipolares instantáneas. 


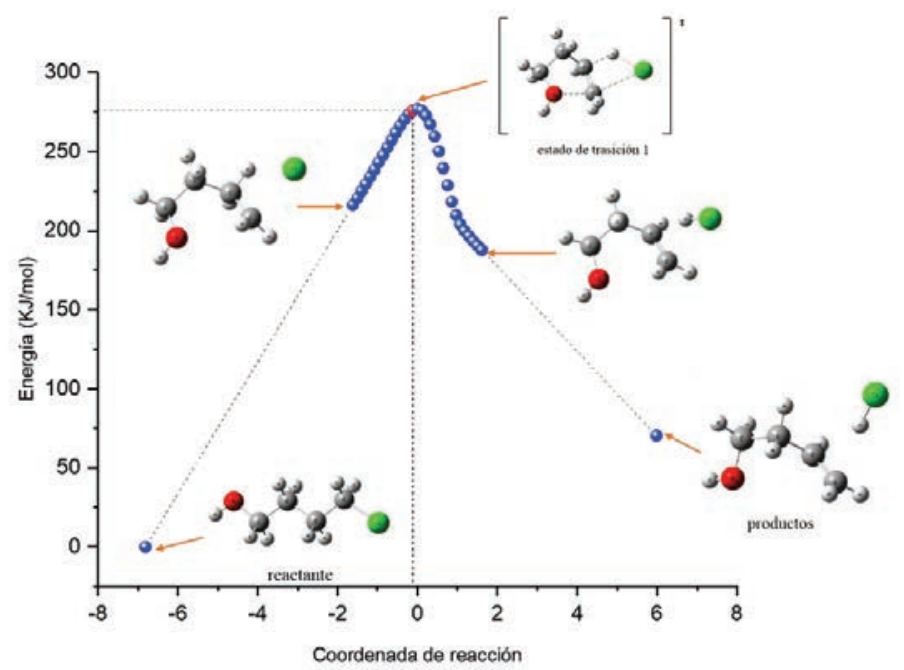

Figura 7. IRC para la primera etapa de la segunda vía de reacción de la pirolisis del 4-cloro-1-butanol empleando el nivel de teoría B3LYP/6-31G(d,p)

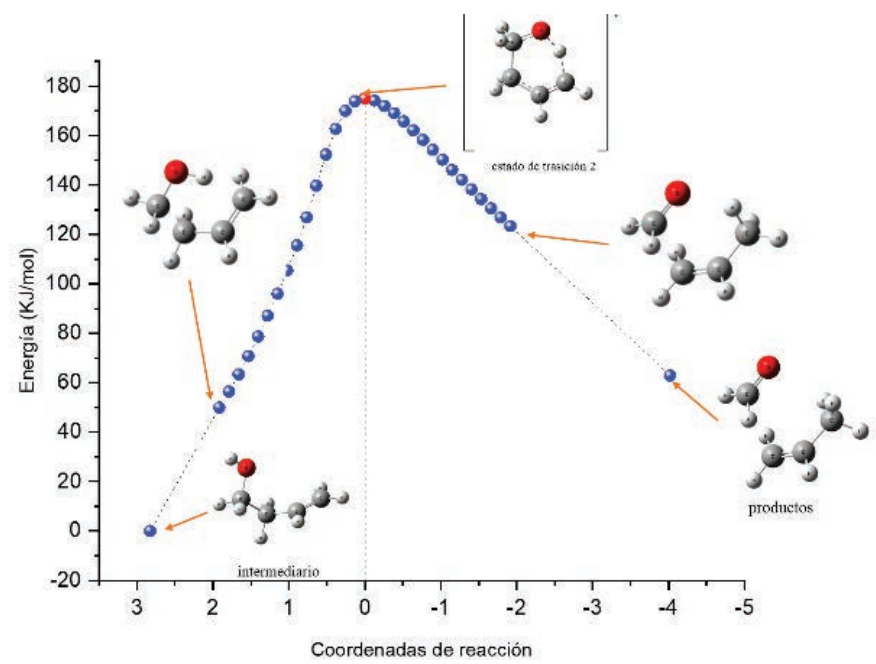

Figura 8. IRC para la segunda etapa de la segunda vía de reacción de la pirolisis del 4-cloro-1-butanol empleando el nivel de teoría WB97XD/6-31G++(d,p) 
Las Tablas 7-9, muestran los índices de Wiberg. Un tipo de orden de enlace que se utiliza para determinar si los procesos mecanístico son de tipo asincrónicos. Ellos se determinaron tanto para el reactante, estado de transición y producto. El funcional fue el WB97XD y utilizando el conjunto base $6-31 \mathrm{G}(\mathrm{d}, \mathrm{p})$.

Tabla 7. Índices de Wiberg del reactivo (R), estado de transición (TS) y producto (P) para vía 1 en reacción de la pirolisis del 4-cloro-1-butanol en fase gaseosa a $440^{\circ} \mathrm{C}$, obtenidos con el nivel de teoría WB97XD/6-31G++(d,p)

\begin{tabular}{lccccc}
\hline & $\mathrm{C}_{1}-\mathrm{Cl}_{13}$ & $\mathrm{C}_{1}-\mathrm{O}_{14}$ & $\mathrm{O}_{14}-\mathrm{H}_{15}$ & $\mathrm{H}_{15}-\mathrm{Cl}_{13}$ & $\mathrm{~S}_{\mathrm{y}}$ \\
\hline $\boldsymbol{B}_{\mathbf{i}}^{\mathrm{R}}$ & 1,0020 & 0,0008 & 0,7446 & 0,0002 & \\
$\boldsymbol{B}_{\mathbf{i}}^{\text {SS }}$ & 0,3317 & 0,2630 & 0,5865 & 0,1461 & 0,6861 \\
$\boldsymbol{B}_{\mathbf{i}}^{\text {P }}$ & 0,0027 & 0,8821 & 0,1052 & 0,8141 & \\
$\boldsymbol{\%}_{\boldsymbol{v}}$ & 67,08 & 29,75 & 24,73 & 17,93 & \\
\hline
\end{tabular}

Tabla 8. Índices de Wiberg del reactivo (R), estado de transición (TS) y producto (P) para primera etapa de la vía 2 en reacción de la pirolisis del 4-cloro-1-butanol en fase gaseosa a $440^{\circ} \mathrm{C}$, obtenidos con el nivel de teoría B3LYP/6-31G(d,p)

\begin{tabular}{lccccc}
\hline & $\mathrm{C}_{1}-\mathrm{Cl}_{13}$ & $\mathrm{C}_{1}-\mathrm{C}_{2}$ & $\mathrm{C}_{2}-\mathrm{H}_{6}$ & $\mathrm{Cl}_{13}-\mathrm{H}_{6}$ & $\mathrm{~S}_{\mathrm{y}}$ \\
\hline $\mathbf{B}_{i}^{\mathrm{R}}$ & 0,9976 & 1,0240 & 0,9070 & 0,0022 & \\
$\mathbf{B}_{i}^{\mathrm{S}}$ & 0,3332 & 1,3690 & 0,4824 & 0,2860 & 0,8341 \\
$\mathbf{B}_{i}^{\mathrm{P}}$ & 0,0183 & 1,9332 & 0,0276 & 0,8694 & \\
$\boldsymbol{\%}_{v}$ & 67,84 & 37,95 & 48,28 & 32,73 & \\
\hline
\end{tabular}

Tabla 9. Índices de Wiberg del reactivo (R), estado de transición (TS) y producto (P) para segunda etapa de la vía 2 en reacción de la pirolisis del 4-cloro-1-butanol en fase gaseosa a $440^{\circ} \mathrm{C}$, obtenidos con el nivel de teoría WB97XD/6-31G++(d,p)

\begin{tabular}{lccccccc}
\hline & $\mathrm{C}_{1}-\mathrm{H}_{13}$ & $\mathrm{H}_{13}-\mathrm{O}_{12}$ & $\mathrm{O}_{12}-\mathrm{C}_{7}$ & $\mathrm{C}_{7}-\mathrm{C}_{5}$ & $\mathrm{C}_{5}-\mathrm{C}_{2}$ & $\mathrm{C}_{2}-\mathrm{C}_{1}$ & $\mathrm{~S}_{\mathrm{y}}$ \\
\hline $\mathbf{B}_{i}^{\mathrm{R}}$ & 0,0002 & 0,7464 & 0,9333 & 1,0035 & 1,0290 & 1,9853 & \\
$\mathbf{B}_{i}^{\mathrm{S}}$ & 0,5466 & 0,2510 & 1,3516 & 0,5192 & 1,4360 & 1,3101 & 0,7490 \\
$\mathbf{B}_{i}^{\mathrm{P}}$ & 0,9071 & 0,0022 & 1,8903 & 0,0104 & 1,9631 & 1,0486 & \\
$\mathbf{\% E}_{v}$ & 60,25 & 66,57 & 43,71 & 43,77 & 43,57 & 72,08 & \\
\hline
\end{tabular}


Finalmente, se muestra la Figura 9, en ella se grafica el gradiente de densidad reducido en función del signo de segundo valor propio del Hessiano por la densidad. En objetivo es detectar las zonas de cambio en la topología de las moléculas de estudio. Esta información es impor- tante, pues nos revelan las interacciones tipo puente hidrógeno, van der Waals y en generar de tipo no covalentes. Los cambios de colores de azul (atracciones) hasta rojo (repulsiones) y verde (no covalentes) ponen en evidencia el mecanismo estudiado.
$\mathbf{R}$

(a)
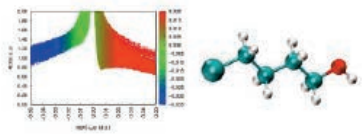

(b)
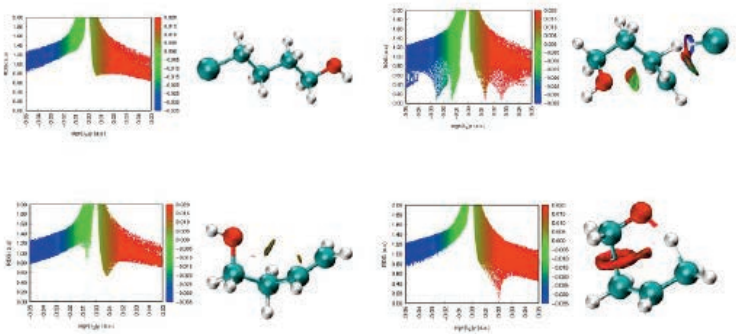

TS
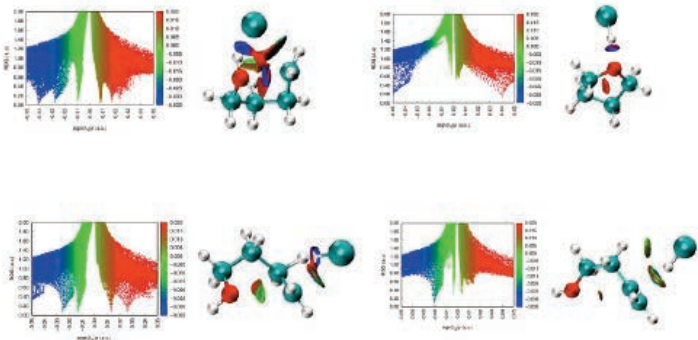

(c)
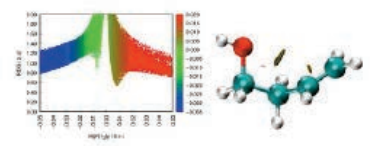
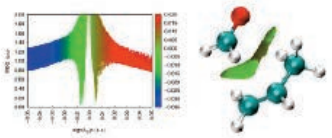

Figura 9. Análisis $\mathrm{NCl}$ para el reactivo (R), estado de transición (TS) y producto (P) de (a) la vía 1, (b) la primera etapa de la vía 2 y (c) la segunda etapa de la vía 2 de la pirolisis de 4-cloro-1-butanol en fase gaseosa a $440{ }^{\circ} \mathrm{C}$

Las Tablas 10-12, muestran los índices IBSI "Intrinsic Bond Strength Index", a través de estos índices se puede correlacionar directamente con la fuerza del enlace en la medida que éste se rompe o forma. Este se calcula al integrar el área obtenida al graficar el índice IGM (dg) versus el signo del segundo valor del Hessiano por la densidad electrónica. 
Tabla 10. Variación del IBSI para las estructuras de la vía 1 en la pirolisis del 4-cloro-1-butanol en fase gaseosa a $440^{\circ} \mathrm{C}$, obtenidos con el nivel de teoría WB97XD/6-31G++(d,p)

\begin{tabular}{lccc}
\hline & \multicolumn{3}{c}{ IBSI } \\
\hline Enlaces & reactivo & TS & producto \\
\hline $\mathrm{C}_{1}-\mathrm{Cl}_{13}$ & 0,675 & 0,072 & 0,009 \\
$\mathrm{C}_{1}-\mathrm{O}_{14}$ & 0,002 & 0,112 & 1,060 \\
$\mathrm{O}_{14}-\mathrm{H}_{15}$ & 1,480 & 1,267 & 0,142 \\
$\mathrm{H}_{15}-\mathrm{Cl}_{13}$ & 0,000 & 0,116 & 0,753 \\
\hline
\end{tabular}

Tabla 11. Variación del IBSI para las estructuras de la primera etapa de vía 2 en la pirolisis del 4-cloro-1-butanol en fase gaseosa a $440^{\circ} \mathrm{C}$, obtenidos con el nivel de teoría B3LYP/6-31G(d,p)

\begin{tabular}{lccc}
\hline & \multicolumn{3}{c}{ IBSI } \\
\hline Enlaces & reactivo & TS & producto \\
\hline $\mathrm{C}_{1}-\mathrm{Cl}_{13}$ & 0,675 & 0,065 & 0,006 \\
$\mathrm{C}_{1}-\mathrm{C}_{2}$ & 0,872 & 1,177 & 1,410 \\
$\mathrm{C}_{2}-\mathrm{H}_{6}$ & 0,901 & 0,596 & 0,046 \\
$\mathrm{H}_{6}-\mathrm{Cl}_{13}$ & 0,010 & 0,200 & 0,782 \\
\hline
\end{tabular}

Tabla 12. Variación del IBSI para las estructuras de la segunda etapa de vía 2 en la pirolisis del 4-cloro-1-butanol en fase gaseosa a $440^{\circ} \mathrm{C}$, obtenidos con el nivel de teoría WB97XD/6-31G++(d,p)

\begin{tabular}{lccc}
\hline & \multicolumn{3}{c}{ IBSI } \\
\hline Enlaces & reactivo & TS & producto \\
\hline $\mathrm{C}_{5}-\mathrm{C}_{7}$ & 0,861 & 0,322 & 0,013 \\
$\mathrm{C}_{7}-\mathrm{O}_{12}$ & 1,102 & 1,702 & 2,066 \\
$\mathrm{O}_{12}-\mathrm{H}_{13}$ & 1,481 & 0,353 & 0,010 \\
$\mathrm{H}_{13}-\mathrm{C}_{1}$ & 0,002 & 0,593 & 0,879 \\
$\mathrm{C}_{1}-\mathrm{C}_{2}$ & 1,436 & 1,125 & 0,909 \\
$\mathrm{C}_{2}-\mathrm{C}_{5}$ & 0,900 & 1,208 & 1,383 \\
\hline
\end{tabular}




\section{DISCUSIÓN}

El mecanismo propuesto para la reacción de descomposición térmica en fase gaseosa del 4-cloro-1-butanol consta de dos vías de reacción. La primera vía de reacción ocurre mediante un estado de transición cíclica concertado de cuatro miembros, dando como productos tetrahidrofurano y cloruro de hidrogeno, según se observa en el esquema 3. La formación de este estado de transición se puede explicar debido a la polaridad de los enlaces $\left.\mathrm{C}-\mathrm{Cl}\left(\mathrm{C}^{\delta+}\right) \ldots \mathrm{Cl}^{\delta_{-}}\right)$y $\mathrm{H}-\mathrm{O}$ $\left(\mathrm{H}^{\delta+} \ldots \mathrm{O}^{\delta-}\right)$ en la reacción de deshidrocloración.

Para la segunda vía de reacción se plantea un mecanismo en dos etapas, con la formación de un estado de transición cíclico concertado de cuatro miembros, con asistencia anquimérica del grupo $\mathrm{OH}$, que da como resultado cloruro de hidrógeno 3-buten-1-ol, este último actúa como intermediario para la segunda etapa formando a su vez un estado de transición cíclico concertado de seis miembros dando los productos formaldehido y propeno, como se observa en el esquema 3.
Los parámetros termodinámicos y cinéticos calculados mediante diversos niveles de teoría para el mecanismo de la vía 1 y las 2 etapas de la vía 2 de la pirolisis del 4-cloro1-butanol se muestran en la tabla 1 , 2 y 3 respectivamente. El nivel de teoría para la vía 1 que dio una energía de activación más cercana a la experimental fue WB97XD/6$31 \mathrm{G}++(\mathrm{d}, \mathrm{p})$, mientras que para la primera y segunda etapa de la vía 2 fueron B3LYP/6-31G(d,p) y WB97XD/6-31G++(d,p), respectivamente.

Los parámetros estructurales de la Tabla 4 muestran que en el estado de transición de la vía 1 ocurre un alargamiento del enlace entre los átomos $\mathrm{Cl}_{13}-C_{1}$, de $1,80 \AA$ a $2,78 \AA$, lo mismo que para $\mathrm{O}_{14}-\mathrm{H}_{15}$ que pasan de 0,96 ̊ a 0,99 A, lo que evidencia la tendencia a la ruptura de estos enlaces para la formación de $\mathrm{HCl}$, mientras que la distancia de los enlaces $\mathrm{C}_{1}-\mathrm{O}_{14}$ pasan de $4,89 \AA$ a 2,30 $\AA$, lo que evidencia la tendencia a la formación de tetrahidrofurano. En la Tabla 5 están los parámetros estructurales para la primera etapa de vía 
2 , donde se observa que en el estado de transición ocurre un alargamiento del enlace entre al pasar de 1,82 a 2,69 $\AA$, igualmente, para el enlace entre los átomos de 1,10 $\AA$ a 1,25 $\AA$, debido a la ruptura de estos enlaces para la formación de $\mathrm{HCl}$, mientras que el enlace entre los átomos pasa de 1,52 $\AA$ a 1,40 $\AA$, lo que indica la tendencia a la formación del intermediario 3-buten-1-ol. Luego en la Tabla 6 se muestran los parámetros estructurales de la vía 2 de reacción, donde observa en el estado de transición un ocurre un distanciamiento entre los átomos $\mathrm{C}_{5}-\mathrm{C}_{7}$, al pasar de $1,52 \AA$ a $1,94 \AA$, lo mismo que en el enlace $\mathrm{O}_{12}-\mathrm{H}_{13}$ de $0,96 \AA$ a 1,40 , debido a la formación de los productos finales formaldehido y propeno. Cabe resaltar que la numeración de los átomos en la segunda etapa de la vía 2 cambia.

Los estados de transición para la vía 1 y las dos etapas de la vía 2 se confirmaron median el cálculo de los gráficos de coordenadas de reacción intrínseca (IRC) como se observa en las Figuras 6-8. En estos gráficos fueron corregidos para tener la energía relativa a los reactivos y así determinar sus coordenadas de reacción. Se calcularon los índices de enlace de Wiberg para estudiar la evolución de la reacción a lo largo de las coordenadas implicadas en la formación de productos. Los índices de Wiberg calculados para la vía 1 se indican en la Tabla 7, mostrando que la reacción de eliminación está dominada por la ruptura del enlace entre los átomos $\mathrm{C}_{1}-\mathrm{Cl}_{13}$, con un $67,08 \%$ de avance. Se observa un menor progreso en los demás enlaces involucrados en la reacción. La reacción presenta una sincronicidad de 0,69, lo que indica un proceso más asincrónico. Los órdenes de enlace para la reacción de la primera etapa de la vía 2 se muestran en la Tabla 8 , se observa un mayor avance entre los enlaces de los átomos $\mathrm{C}_{1}-\mathrm{Cl}_{13}$ y $\mathrm{C}_{2}$ $\mathrm{H}_{6}$ con $67,84 \%$ y $48,28 \%$ respectivamente, lo que demuestra que la reacción está dominada por la ruptura de estos enlaces. La sincronicidad para esta reacción fue de 0,83 , lo que indica que el proceso más sincrónico.

Los órdenes de enlace para la reacción de la segunda etapa de la vía 2 se muestran en la Tabla 9, donde se observa que el mayor avance de la reacción se da para el enlace $\mathrm{C}_{2}$ $\mathrm{C}_{1}$ con $72,08 \%$ de avance, además se observa el cambio en el orden 
entre los enlaces $\mathrm{C}_{1}-\mathrm{H}_{13}$ y $\mathrm{H}_{13}-\mathrm{O}_{12}$ indica la transferencia del hidrógeno del $\mathrm{O}_{12}$ al carbono $\mathrm{C}_{1}$. La sincronicidad para esta reacción fue de 0,75, lo que indica un proceso moderadamente sincrónico

El análisis de la topología molecular de interacciones no covalentes $(\mathrm{NCl})$ para las estructuras de la via 1, la primera y segunda etapa de la vía 2 del mecanismo de reacción de la pirolisis del 4-cloro-1-butanol se muestran en la Figura 9. En la estructura del estado de transición para la vía 1 se observa una interacción de atracción en el $\mathrm{H}_{15}$ y $\mathrm{Cl}_{13}$, igualmente entre el $\mathrm{O}_{14}-\mathrm{C}_{1}$, debido a la formación de enlaces.

Para el estado de transición de la primera etapa de la vía 2 se observa una interacción no covalente entre el $\mathrm{O}_{14}$ y $\mathrm{C}_{1}$, lo que evidencia la asistencia anquimérica del oxígeno en la eliminación del $\mathrm{Cl}_{13}$ como $\mathrm{HCl}$.

Para el estado de transición de la segunda etapa de la vía 2 se observa una fuerte repulsión entre el $\mathrm{C}_{5} \mathrm{yC}_{7}$, debido a la tendencia para la formación de los productos finales formaldehido y propeno.
Usando el índice de fuerza de enlace intrínseco (IBSI) en las estructuras de la vía 1 , según se muestra en la Tabla 10, se observa una disminución en la fuerza del enlace $\mathrm{C}_{1}-\mathrm{Cl}_{13}$ al igual el enlace $\mathrm{O}_{14}-\mathrm{H}_{15}$ llegando al rango no covalente, debido a la ruptura de estos enlaces, mientras se da un aumento de la fuerza del enlace $\mathrm{H}_{15}-\mathrm{Cl}_{13}$ llegando al rango de enlace covalente, debido a la formación de $\mathrm{HCl}$.

La variación del IBSI en las estructuras de la primera etapa de la vía 2, como se muestra en la Tabla 11, indican la disminución en la fuerza de los enlaces $\mathrm{C}_{1}-\mathrm{Cl}_{13}$ y $\mathrm{C}_{2}-\mathrm{H}_{6}$ Ilegando al rango no covalente, debido a la ruptura de estos enlaces, así mismo se da un incremento de la fuerza de enlace entre $\mathrm{H}_{6}-\mathrm{Cl}_{13}$, debido a la formación de $\mathrm{HCl}$, y de $\mathrm{C}_{1}-\mathrm{C}_{2}$ por la formación de un doble enlace, ambos llegando al rango covalente. Para las estructuras de la segunda etapa de la vía 2 la variación del IBSI, según la Tabla 12, se da una disminución de los enlaces $\mathrm{C}_{5}-\mathrm{C}_{7}$ y $\mathrm{O}_{12}-\mathrm{H}_{13}$, ambos Ilegando al rango no covalente, debido a la ruptura de los enlaces por la formación de los productos finales formaldehido $y$ propeno. 


\section{CONCLUSIÓN}

El mecanismo de reacción para la pirolisis del 4-cloro-1-butanol en fase gaseosa, se estudió mediante la teoría del funcional de la densidad, teniendo dos vías de reacción. En la primera vía se da mediante un estado de transición cíclico de cuatro miembros y la segunda vía ocurre en dos etapas, la primera mediante un estado de transición cíclico de cuatro miembros y la segunda etapa con un estado de transición cíclico de seis miembros. Se usaron diversos niveles de teoría para optimizar las verificaron las estructuras de los estados de transición planteados me- diante cálculos IRC. Los parámetros termodinámicos y cinéticos se calcularon para una serie de funcionales que incluían dispersiones empíricas y sus valores se contrastaron con sus pares experimentales. Se obtuvieron buenos resultados con los niveles de teoría WB97XD/6-31G++(d,p), para la primera vía de reacción, B3LYP/6$31 \mathrm{G}(\mathrm{d}, \mathrm{p})$ y WB97XD/6-31G++(d,p), para la primera y segunda etapa de la segunda vía respectivamente. Los índices de enlace, así como la variación de la fuerza de enlace, son consistentes con los mecanismos planteados.

\section{AGRADECIMIENTOS}

OLGT agradece a la Universidad Nacional de San Marcos, por permitirme hacer este trabajo durante los cursos de Introducción a la Investigación A y B que ofrece la Facultad de Química e Ingeniería Química.

\section{LISTA DE REFERENCIAS}

Contreras-García, J., Johnson, E. R., Keinan, S., Chaudret, R., Piquemal, J.-P., Beratan, D. N., \& Yang, W. (2011). NCIPLOT: A Program for Plotting Noncovalent Interaction Regions. Journal of Chemical Theory and Computation, 7(3), 625-632.

Chuchani G. (1995). The Chemistry of Halides, Pseudo-Halides and Azides, (Chapter

19), E.S. Patai and Z. Rapopport, eds, Wiley, New York, 1069. 
Chuchani G. and Martín I. (1986). The elimination kinetics of methoxyalkyl chlorides in the gas phase. Evidence for neighboring group participation. J. Phys. Chem., $90,431$.

Chuchani, G., R.M. \& Martín, I. (1987). Neighboring Group Participation in the Pyrolysis Kinetics of 4-Chloro-1-Butanol in the Gas Phase, Instituto Venezolano de Investigaciones científicas (I.V.I.C.).

Foresman B.J. \& Frish $Æ$ E. (1996). Exploring chemistry with electronic methods, 2nd, Edition Gaussian Inc Pittsburg PA.

Gaussian NBO version 16., incluída en Gaussian 16.

Gaussian 16, Revision C.01, M. J. Frisch, G. W. Trucks, H. B. Schlegel, G. E. Scuseria, M. A. Robb, J. R. Cheeseman, G. Scalmani, V. Barone, G. A. Petersson, H. Nakatsuji, X. Li, M. Caricato, A. V. Marenich, J. Bloino, B. G. Janesko, R. Gomperts, B. Mennucci, H. P. Hratchian, J. V. Ortiz, A. F. Izmaylov, J. L. Sonnenberg, D. WiIliams-Young, F. Ding, F. Lipparini, F. Egidi, J. Goings, B. Peng, A. Petrone, T. Henderson, D. Ranasinghe, V. G. Zakrzewski, J. Gao, N. Rega, G. Zheng, W. Liang, M. Hada, M. Ehara, K. Toyota, R. Fukuda, J. Hasegawa, M. Ishida, T. Nakajima, Y. Honda, O. Kitao, H. Nakai, T. Vreven, K. Throssell, J. A. Montgomery, Jr., J. E. Peralta, F. Ogliaro, M. J. Bearpark, J. J. Heyd, E. N. Brothers, K. N. Kudin, V. N. Staroverov, T. A. Keith, R. Kobayashi, J. Normand, K. Raghavachari, A. P. Rendell, J. C. Burant, S. S. Iyengar, J. Tomasi, M. Cossi, J. M. Millam, M. Klene, C. Adamo, R. Cammi, J. W. Ochterski, R. L. Martin, K. Morokuma, O. Farkas, J. B. Foresman, and D. J. Fox. (2016). Gaussian, Inc., Wallingford CT.

Klein J., Khartabil H, Boisson, J.-C., Contreras-García, J., Piquemal J.-P. \& Hénon E. (2020), New Way for Probing Bond Strength. J. Phys. Chem. A, 124, 1850-1860.

Maccoll A. (1969). Heterolysis and the pyrolysis of alkyl halides in the gas phase. Chem Rev., 69, 33.

Maccoll A. \& Thomas P.J. (1955). A correlation between the rates of unimolecular pyrolysis of alkyl halides in the gaseous phase and sni rates in solution. Nature 176, 392. 
Maccoll A. \& Thomas P. J. (1967). Molecular reactions in the gas phase and the quasiheterolytic hypothesis. Prog. React Kinet., 4, 119.

Moyano A., Pericá M.A. \& Valenti E. (1989). A theoretical study on the mechanism of the thermal and the acid-catalyzed decarboxylation of 2-oxetones ( $\beta$-lactones). J Org Chem., 54, 573

Smith G.G. \& Kelly F.W. (1971). Structure relationships in homogeneous gas-phase reactions: thermolyses and rearrangements. Progr. React. Kinet., 8, 75.

Scale factors in http: //cccbdb.nist.gov/vibscalejust.asp, (b) Database of Frequency Scaling Factors for Electronic Structure Methods https: //comp.chem.umn.edu/freqscale/index.html.

Tian L. \& Feiwu Ch. (2012). Multiwfn: A Multifunctional Wavefunction Analyzer, J. Comput. Chem., 33, 580-592.

VMD - Visual Molecular Dynamics [Internet]. Ks.uiuc.edu. 2021.

Wiberg K.B., (1968). Application of the Pople-Santry-Segal complete neglect of differential overlap method to the cyclopropyl-carbinyl and cyclobutyl cation and to bicyclobutane. Tetrahedron. 24, 1083-1096. 